\title{
HORN CLASSES AND REDUCED DIRECT PRODUCTS
}

\author{
BY
}

RICHARD MANSFIELD

ABSTRACT. Boolean-valued model theory is used to give a direct proof that an $E C_{\Delta}$ model class closed under reduced direct products can be characterized by a set of Horn sentences. Previous proofs by Keisler and Galvin used either the G. C. H. or involved axiomatic set theory.

We shall give a direct proof of the theorem that an $E C_{\Delta}$ model class is closed under reduced direct products iff it is characterizable by a set of Horn sentences. This was first proven by Keisler as a consequence of the continuum hypothesis. Galvin then proved that in ZF set theory it is provably equivalent to a certain arithmetical statement. From these two results, it follows that the theorem is true in the constructible universe for set theory and is consequently true. This indirect proof of a simple proposition of model the ory seems overly ornate. We shall carry out the main features of Keisler's argument within the system developed in [3] and prove the theorem without any use of axiomatic set theory. Subsequent to this proof Shelah has given another direct proof of this same theorem [5]. His methods do not use Boolean-valued models as do mine, but rather closely follow his proof that elementarily equivalent models have isomorphic ultrapowers.

1. A major tool in our proof is the theory of first order Boolean-valued models. Since the standard notation for model theory becomes cumbersome in the Boolean case, we give an alternate system; a model is identified with its truth function. For $\mathfrak{Q}$ a finitary language without function symbols, an $\mathfrak{E}$ structure is a set of constant symbols $|\mathfrak{U}|$ containing all the constant symbols of $\mathfrak{L}$ together with a function $\mathfrak{U}$ from $\mathfrak{L}(|\mathfrak{U}|)$ into a complete Boolean algebra satisfying the conditions:

1. $\mathscr{U}(a=b)=1$,

2. $\mathscr{U}(a=b) \leq \mathscr{U}(b=a)$,

3. $\mathfrak{U}(a=b) \wedge \mathfrak{U}(b=c) \leq \mathfrak{U}(a=c)$,

4. For $\phi$ an atomic sentence, $\mathscr{U}(\phi(a)) \wedge \mathfrak{U}(a=b) \leq \mathfrak{U}(\phi(b))$,

5. $\mathscr{U}(\phi \vee \psi)=\mathscr{U}(\phi) \vee \mathfrak{U}(\psi)$,

6. $\mathscr{U}(\neg \phi)=\neg \mathfrak{U}(\phi)$,

7. $\mathscr{U}[\exists x \phi(x)]=V_{a \in|x|} \mathfrak{R}[\phi(a)]$.

Received by the editors January 15,1971 .

AMS (MOS) subject classifications (1969). Primary 1250; Secondary 0242. 
When the language $\mathcal{Q}$ contains function symbols, these must first be interpreted by actual functions from the appropriate powers of $|\mathfrak{Q}|$ into $|\mathfrak{U}|$ before proceeding as above. An $\mathcal{Q}$-structure satisfies the maximum principle if the truth value of any existential statement is always equal to the truth value of some instance. Any $\mathcal{L}$-structure has a canonical elementary extension satisfying the maximum principle.

Various basic operations of model theory can be generalized to Boolean model theory. If $\left\{\mathscr{Q}_{i}\right\}_{i \in I}$ is a collection of $\mathcal{Q}$-structures with corresponding algebras $\left\{B_{i}\right\}_{i \in I}, \Pi_{i} \mathfrak{U}_{i}$ can be defined as a $\Pi_{i} B_{i}$-valued model. The set of constant symbols for $\Pi_{i} \mathscr{U}_{i}$ is just the usual cartesian product of the component symbols and truth is defined by the equation

$$
\prod \mathscr{U}_{i}\left[\phi\left(\left\langle a_{i}\right\rangle_{i \in I}\right)\right]=\left\langle\mathscr{U}_{i}\left[\phi\left(a_{i}\right)\right]\right\rangle_{i \in I} \text {. }
$$

This definition should actually be called the covariant direct product. It has the drawback that it does not specialize to the traditional definition in the twovalued case; the product of a pair of two-valued models is a four-valued model. The traditional definition will be a special case of our definition of a reduced direct product. The contravariant direct product, which is defined using the algebra $\Sigma_{i} B_{i}$, does not have this drawback and has a much better claim to the name "direct product." However it is the covariant product which is useful for the purposes of this paper.

The Boolean power of a two-valued model is the structure that was used in the construction of Boolean ultrapowers in [3]. For $\mathscr{U}$ a two-valued model and $B$ a complete Boolean algebra the $B$-valued power $\mathscr{U}^{(B)}$ is defined as follows. The constant symbols for $\mathfrak{U}^{(B)}$ is the set of all functions from the constants of $\mathcal{U}$ into $B$ whose ranges partition $B$, i.e.

$$
\left\{f \in B^{\mathfrak{a}}: a_{1} \neq a_{2} \rightarrow f\left(a_{1}\right) \wedge f\left(a_{2}\right)=0 \text { and } \bigvee_{a} f(a)=1\right\} \text {. }
$$

Truth is defined by the equation

$$
\mathfrak{Y}^{(B)}\left[\phi\left(f_{1} \cdots f_{n}\right)\right]=\bigvee\left\{\bigwedge_{i=1}^{n} f_{i}\left(a_{i}\right): \mathscr{U} \vDash \phi\left(a_{1}, \cdots, a_{n}\right)\right\} \text {. }
$$

For a more extensive treatment of this structure the reader is referred to $\left[3, \S_{1}\right]$ where it is discussed in necessary detail. In [3] it is shown that $\mathscr{O}^{(B)}$ is an elementary extension of $\mathfrak{U}$. Again our definition does not specialize to the traditional one when $B$ is a power set algebra; we will need to first reduce by a filter.

If $A$ and $B$ are both complete Boolean algebras, we define an $A$-valued filter on $B$ to be a function $D$ from $B$ into $A$ such that $D\left(b_{1} \wedge b_{2}\right)=$ 
$D\left(b_{1}\right) \wedge D\left(b_{2}\right)$ and $D(1)=1$. If in addition $D(\neg b)=\neg D(b), D$ is an ultrafilter. $D$ is proper if $D(0)=0$. A function $E$ from $B$ into $A$ has the finite intersection property if $E(0)=0$ and also $X_{i=1}^{n} b_{i}=0$ implies $X_{i=1}^{n} E\left(b_{i}\right)=0$. Just as with two-valued filters any function with the finite intersection property uniquely generates a filter. This is accomplished by the definition

$$
D(b)=\bigvee\left\{\bigwedge_{i=1}^{n} E\left(b_{i}\right): \prod_{i=1}^{n} b_{i} \leq b\right\}
$$

If $\left\{\mathscr{U}_{i}\right\}_{i \in I}$ is a collection of $\mathcal{L}$-structures satisfying the maximum principle and $D$ is a $B$-valued filter on the product algebra, we define the Boolean reduced product $\Pi_{i} \mathscr{O}_{i} / D$ as a $B$-valued model. The set of constant symbols is the same as in $\Pi \mathscr{O}_{i}$ and truth for atomic $\phi$ is defined by

$$
\prod \mathscr{N}_{i} / D(\phi)=D\left[\prod \mathscr{U}_{i}(\phi)\right] \text {. }
$$

Truth for arbitrary sentences is then defined by induction according to conditions $5,6,7$ of the definition of an $\mathcal{L}$-structure.

In the special case that $D$ is an ultrafilter, it can be shown that, for arbitrary $\phi, \Pi \mathscr{U}_{i} / D(\phi)=D\left[\Pi \mathscr{U}_{i}(\phi)\right]$ but in the general case this is not so [3]. However, when $\phi$ is a Horn sentence it is an easy exercise to prove that

$$
\prod \mathscr{U}_{i} / D(\phi) \geq D\left[\Pi \mathfrak{U}_{i}(\phi)\right] \text {. }
$$

This shows that Horn sentences are preserved by reduced direct products.

Since we are allowing the use of Boolean-valued models, nontrivial use of the above definition can be made even when only one model is involved. $\mathfrak{U}^{(B)} / D$, the application of the above definition to just the one model $\mathfrak{U}^{(B)}$, is a reduced direct power of the two-valued model $\mathscr{O}$. When $D$ is a two-valued ultrafilter, this structure is just the Boolean ultrapower studied in [3].

If $D$ is a two-valued filter on $2^{I}$ and each $\mathscr{U}_{i}$ is a two-valued model, $\Pi \mathscr{A}_{i} / D$ is the traditional reduced direct product of the $\mathscr{U}_{i}$ 's. If $D$ is the trivial filter $\{1\}, \Pi \mathscr{U}_{i} / D$ is just the traditional cartesian product and $\mathfrak{U}^{\left(2^{I}\right)} / D$ is canonically isomorphic to the traditional cartesian power of $\mathscr{U}$.

We shall conclude this section by stating a lemma which shall be used in the main argument. This lemma follows easily from

Theorem 1.1. If $\mathscr{O}$ is a B-valued $\mathcal{Q}$-structure and $B$ satisfies the $<\boldsymbol{\aleph}_{1}, \infty$ distribution law and $\mathcal{L}$ is countable, $\mathcal{U}$ bas a countable substructure $B$ for which there is a nonzero $b$ in $B$ with

$$
\mathfrak{U}(\phi) \wedge b=\mathfrak{B}(\phi) \wedge b
$$

for every $\phi$ in $\mathscr{L}(\Re)$. 
Since this paper is not meant to be a treatise on Boolean-valued model theory, we are leaving the proof of this theorem to the reader. Very briefly, in order to prove it one must first pass to a certain elementary extension $\mathfrak{U}^{\prime}$ of $\mathfrak{U}$ in which the truth value of any existential statement is equal to the truth value of one of its instances [4]. In the extension the Löwenheim-Skölem argument can be applied exactly as in two-valued logic. Since the extension $I$ have in mind satisfies the condition that, for any $\left.a^{\prime} \in\right|^{\mathfrak{X}^{\prime}} \mid, \bigvee_{a \in|\mathfrak{x}|^{2 \mathfrak{I}^{\prime}}\left(a=a^{\prime}\right)=1 \text {, the }}$ distributivity law produces the desired element $b$ and countable structure $B$.

Lemma 1.2. If $\left\{\mathscr{U}_{i}\right\}_{i \in I}$ is a collection of two-valued models and $D$ is a $B$ valued filter on $2^{I}$ and $B$ satisfies the $\left\langle\kappa_{1}, \infty\right.$ distribution law, there is a two-valued ultrafilter $\mu$ on $B$ such that for any sentence $\phi$ without parameters

$$
\Pi \mathfrak{U}_{i} / D \circ \mu(\phi)=\mu\left[\Pi \mathfrak{U}_{i} / D(\phi)\right] .
$$

Proof. There is a countable $\mathscr{Z} \subseteq \Pi_{\mathcal{U}_{i}} / D$ and an element $b \in B$ satisfying Theorem 1.1. Let $\mu$ be an ultrafilter on $B$ containing $b$ and preserving all of the countably many sups used to evaluate sentences in $\mathscr{L}(\mathfrak{B})$. The existence of such a $\mu$ is guaranteed by the Rasiowa-Sikorski homomorphism theorem. $\mu$ is easily seen to satisfy the lemma.

2.

Theorem 2.1. If $K$ is a model class closed under elementary equivalence and reduced direct products, $K$ can be characterized by a set of Horn sentences.

We stress that despite all the Boolean constructions of the previous section this is a two-valued theorem; the models in $K$ are two-valued and the reduced products are the traditional ones. The proof, however, will be quite Boolean. What we shall actually prove is that any model for the Horn theory of $K$ is elementarily equivalent to a reduced product of $K$, and hence $K$ can be characterized by its Horn theory.

For the sake of completeness we give a definition of the class of Horn formulae. A basic Horn formula is a formula in the form $X_{i=1}^{n} \phi_{i} \rightarrow \phi_{0}$ where each of the $\phi_{i}$ for $0 \leq i \leq n$ is atomic (true and false are counted as atomic sentences). A Horn formula is a formula in prenex normal form whose matrix is a conjunction of basic Horn formulae. The Horn theory of $K$ is the set of Horn sentences true in every member of $K$.

Let $\mathscr{B}$ be a model for the Horn theory of $K$. By taking an elementary extension if necessary we may assume that $B$ is $K_{1}$-saturated. (For a definition of $\boldsymbol{N}_{1}$-saturation, see $[2, \mathrm{p} .310]$.) Let $\left\{\mathcal{U}_{i}\right\}_{i \in I}$ be an indexed collection from $K$ such 
that any Horn sentence true in all but finitely many $\mathscr{C}_{i}$ is also true in $\mathscr{B}$. Such a collection can easily be constructed since a Horn sentence false in $B$ must also be false in at least one element of $K$ and this element can be included infinitely many times in the collection.

We let the notation $f: \Pi \mathscr{U}_{i} \rightarrow \mathfrak{B}$ mean that $f$ is a partial function from $\Pi \mathscr{U}{ }_{i}$ into $B$ such that whenever the Horn sentence $\phi\left(a_{1}, \ldots, a_{n}\right)$ is true in all but finitely many $\mathscr{U}_{i}$ and $\left\{a_{1}, \ldots, a_{n}\right\} \subseteq \operatorname{dom} f, \phi\left[f\left(a_{1}\right), \ldots, f\left(a_{n}\right)\right]$ is true in $\mathscr{P}$. (Sometimes when the parameters of $\phi$ are not explicitly listed, we shall use the notation $f(\phi)$ for the image formula.) Our first step is to construct a certain Boolean algebra. This will be done by using the regular open subsets of a topological space. Let $T$ be

$$
\left\{f: \prod \mathscr{U}_{i} \rightarrow \mathfrak{B} \text { and }|f|=\aleph_{1}\right\}
$$

Each countable $Q: \Pi \mathfrak{H}_{i} \rightarrow B$ defines a subset of $T$, namely $[Q]=\{f \in T: Q \subseteq f\}$. We give $T$ the topology generated by the $[Q]$ 's, and let $B$ be the regular open algebra of that topology. In order to show that $B$ is nontrivial we must prove that $T$ is nonempty.

Lemma 2.2. If $Q: \Pi \mathscr{X} \rightarrow \mathfrak{B}$ is countable, there is an $f \in[Q]$ with $a \in \operatorname{dom} f$ and $b \in \mathrm{rng} f$ for any $a$ in $\Pi \mathscr{U}_{i}$ and $b \in \mathbb{R}$.

Proof. The discerning reader will realize that this lemma exactly corresponds to Ke isler's lemma [2, Theorem 3.1]; not surprisingly it has the same proof. We first find a countable $Q_{0}: \Pi \mathscr{U}_{i} \rightarrow \Re$ extending $Q$ with $a \in \operatorname{dom} Q_{0}$. Let $\Gamma$ be the set of Horn formulae with one free variable and parameters from $\operatorname{dom} Q$ such that for all but finitely many $i, \mathfrak{O}_{i} \models \phi[a(i)]$. Then for $\Delta$ a finite subset of $\Gamma$ the sentence $\exists x \quad \mathbb{} \Delta$ is true in all but finitely many $\mathscr{U}_{i}$ and is a Horn sentence. Thus $Q(\exists x M \Delta)$ is true in $B$, i.e., $Q(\Gamma)$ is finitely satisfiable in $B$. Therefore, by the $\boldsymbol{N}_{1}$-saturation of $\mathscr{B}$, there is a $b^{\prime}$ in $\mathscr{B}$ which satisfies every formula in $Q(\Gamma)$. Clearly $Q \cup\left\{\left\langle a, b^{\prime}\right\rangle\right\}$ is the desired extension.

We will now use a parallel argument to find a $Q_{1}: \Pi \mathscr{U}_{i} \rightarrow \mathfrak{P}$ extending $Q_{0}$ with $b \in \operatorname{rng} Q_{1}$. This time let $\Gamma$ be the set of Horn formulae $\phi(x)$ with one free variable and parameters from $\operatorname{dom} Q_{0}$ such that $Q_{0}[\phi(b)]$ is false in $B$. For each $\phi$ in $\Gamma$, let $I_{\phi}=\left\{i: \mathscr{U}_{i} \vDash \exists x \neg \phi(x)\right\}$. Since $Q_{0}[\phi(b)]$ is false, $I_{\phi}$ is infinite. Therefore, by a lemma of Keisler [2, Lemma 1.3], there is a pairwise disjoint collection $\left\{J_{\phi}\right\}_{\phi \in \Gamma}$ of infinite sets with $J_{\phi} \subseteq I_{\phi}$ for each $\phi$ in $\Gamma$. Now pick $a^{\prime}$ in $\Pi \mathscr{U}_{i}$ such that $i \in J_{\phi}$ implies $\mathscr{U}_{i} \models \neg \phi\left[a^{\prime}(i)\right]$. Then $Q_{0} \cup\left\{\left\langle a^{\prime}, b\right\rangle\right\}$ is the desired extension.

We finaily show that $Q_{1}$ can be extended to an element of $T$. Since $\Pi \mathscr{Q}{ }_{i}$ is 
uncountable, we have just shown that any countable $Q: \Pi \mathscr{U}_{i} \rightarrow \mathscr{P}$ can be properly extended; thus a canonical use of Zorn's Lemma gives the desired result.

For $a \in \Pi \mathscr{O}_{i}$ and $b \in \mathfrak{B}$, define

$$
(a, b)=\text { interior (closure }(\{f \in T: f(a)=b\})) \text {. }
$$

Then $(a, b)$ is a regular open subset of $T$ and hence is a member of $B$. Note that $[Q] \subseteq(a, b)$ implies $Q \cup\{\langle a, b\rangle\}: \Pi \mathscr{2} \rightarrow \mathbb{B}$. We can now define a function $j$ from $\Pi \mathscr{U}_{i}$ into $\mathscr{Q}^{(B)}$ by $j(a)(b)=(a, b)$. We must first show that, for each $a$, $j(a)$ is actually a member of $\mathfrak{B}^{(B)}$. Clearly, for $b_{1} \neq b_{2}, j(a)\left(b_{1}\right) \wedge j(a)\left(b_{2}\right)=0$. Suppose that $\bigvee_{b} j(a)(b)<1$. Then there would be a countable $Q: \Pi \mathscr{A}_{i} \rightarrow \mathfrak{B}$ with $[Q] \wedge \bigvee_{b}(a, b)=0$. We have just shown that there is a $Q_{0}$ extending $Q$ with $a$ $\epsilon \operatorname{dom} Q_{0}$. Then,

$$
0<\left[Q_{0}\right] \leq\left[\left\{\left\langle a, Q_{0}(a)\right\rangle\right\}\right] \leq\left(a, Q_{0}(a)\right) \leq \underset{b}{\bigvee_{b}}(a, b) .
$$

In similar fashion it can be shown that $\bigvee_{a}(a, b)=1$.

Lemma 2.3. If $\phi$ is a Horn sentence with parameters from $\Pi \mathscr{U}_{i}$ and $\left\{i: \mathscr{U}_{i} \vDash\right.$ $\phi\}$ is cofinite, $\mathbb{B}^{(B)}[j(\phi)]=1$.

Proof. If $a_{1}, \ldots, a_{n}$ are all the parameters of $\phi$ and $Q: \Pi \mathscr{U}_{i} \rightarrow \mathscr{B}$ and $\left\{a_{1}, \ldots, a_{n}\right\} \subseteq \operatorname{dom} Q$, then $B \models \phi\left[Q\left(a_{1}\right), \ldots, Q\left(a_{n}\right)\right]$. Therefore

$$
\bigwedge_{i=1}^{n} j\left(a_{i}\right)\left(b_{i}\right)>0 \quad \text { implies } B \vDash \phi\left(b_{1}, \cdots, b_{n}\right) .
$$

Consequently,

$$
\phi\left(b_{1}, \cdots, b_{n}\right) \bigwedge_{i=1}^{n} j\left(a_{i}\right)\left(b_{i}\right)=\underset{b_{1} \cdots b_{n}}{\bigvee} M_{i=1}^{n} j\left(a_{i}\right)\left(b_{i}\right)=1
$$

but L. H. S. is $\mathfrak{B}^{(B)}[j(\phi)]$.

Now let $B^{\prime}=\operatorname{rng} j$.

Lemma 2.4. For every $h$ in $B^{(B)}$,

$$
\bigvee\left\{B^{(B)}(h=f): f \in B^{\prime}\right\}=1
$$

Proof. Suppose otherwise; then there is a $Q$ with $[Q] \wedge \bigvee\left\{\mathscr{B}^{(B)}(h=f): f \in \mathscr{B}^{\prime}\right\}=0$. Since $\bigvee_{b} b(b)=1$ there is a $b$ in $B$ with $[Q) \wedge b(b)>0$. Then since $\bigvee_{a}(a, b)=1$ there is an $a$ with $Q \wedge h(b) \wedge(a, b)>0$. But $h(b) \wedge(a, b) \leq$ $\mathfrak{B}^{(\stackrel{a}{B})}[h=j(a)]$.

In $[3, \S 1]$ it was shown that $B^{(B)}$ is an elementary extension of $B$, i.e., a sentence is true in $B$ iff it has value one in $\mathscr{B}^{(B)}$. We now use Lemma 2.4 to show that $B^{(B)}$ is elementarily equivalent to $B^{\prime}$. 


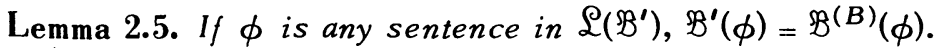

Proof. We proceed by induction on the logical depth of $\phi$. The lemma is true by definition for atomic formula. For negations and disjunctions it follows instantly from the inductive hypothesis without any use of Lemma 2.4. So we assume $\phi=\exists x \psi(x)$. Then

$$
B^{\prime}(\phi)=\bigvee_{f \in \mathscr{B}^{\prime}} B^{\prime}(\psi(f)) \leq \bigvee_{f \in \mathscr{B}^{(B)}} B^{(B)}(\psi(f))=B^{(B)}(\phi)
$$

We must show that the reverse inequality also holds. For each $f$ in $\mathfrak{B}^{\prime}$ and $h$ in $\mathfrak{B}^{(B)}, \mathfrak{B}^{(B)}(\psi(h) \wedge f=h) \leq \mathbb{B}^{(B)}(\psi(f))$. Therefore,

$$
\bigvee_{f \in \mathfrak{B}^{\prime}} \mathbb{B}^{(B)}(\psi(h)) \wedge \mathfrak{B}^{(B)}(f=h) \leq \mathbb{B}^{\prime}(\phi) .
$$

Thus for each $h$ in $\mathfrak{B}^{(B)}, \mathfrak{B}^{(B)}(\psi(h)) \leq \mathfrak{B}^{\prime}(\phi)$ and taking the sup over $h$ gives the desired result.

We now define a $B$-valued filter on $2^{I}$. For each atomic $\phi$ in $\mathscr{L}\left(\Pi \mathscr{U}_{i}\right)$, let $I_{\phi}=\left\{i: \mathscr{U}_{i} \vDash \phi\right\}$. Then let $E\left(I_{\phi}\right)=\mathfrak{B}^{\prime}(j(\phi)) ; E(J)=0$ for any $J \subseteq I$ which is not an $I_{\phi}$. It is straightforward to show using the technique of the next lemma that $E$ has the finite intersection property and thus generates a proper $B$-valued filter $D$.

Lemma 2.6. $j$ is an isomorphism from $\Pi \mathscr{N}_{i} / D$ onto $\mathfrak{R}^{\prime}$.

Proof. We show that, for any atomic sentence,

$$
\prod \mathscr{2}_{i} / D\left(\phi\left(a_{1}, \cdots, a_{n}\right)\right)=B^{\prime}\left(\phi\left(j\left(a_{1}\right), \cdots, j\left(a_{n}\right)\right)\right) .
$$

From the definition of $E$ and $D$ it follows that

$$
\begin{aligned}
& \prod \mathscr{U}_{i} / D\left(\phi\left(a_{1}, \cdots, a_{n}\right)\right)=D\left(\left\{i: \mathscr{U}_{i} \vDash \phi\left(a_{1}(i), \cdots, a_{n}(i)\right)\right\}\right) \\
& \quad \geq E\left(\left\{i: \mathfrak{U}_{i} \vDash \phi\left(a_{1}(i), \cdots, a_{n}(i)\right)\right\}\right)=B^{\prime}\left(\phi\left(j\left(a_{1}\right), \cdots, j\left(a_{n}\right)\right)\right) .
\end{aligned}
$$

In order to prove that equality holds suppose $\left\{\phi_{k}\right\}_{k=1}^{n}$ is a finite set of atomic sentences in $\mathfrak{L}\left(\Pi \mathfrak{U}_{i}\right)$ with $\left\{i: \mathfrak{U}_{i} \vDash \mathbb{X}_{k=1}^{n} \phi_{k}\right\} \subseteq\left\{i: \mathfrak{U}_{i} \models \phi\right\}$. Then for every $i, \mathfrak{O U}_{i} \vDash \mathbb{M}_{k=1}^{n} \phi_{k} \rightarrow \phi$ and this is a Horn sentence; thus by Lemma 2.3 it is valid in $\mathscr{B}^{\prime}$, i.e., $\bigcap_{k=1}^{n} I_{\phi_{k}} \subseteq I_{\phi}$ implies $X_{i=1}^{k} E\left(I_{\phi_{k}}\right)<E\left(I_{\phi}\right)$ and thus $E\left(I_{\phi}\right)$ $=D\left(I_{\phi}\right)$.

Lemma 2.7. B satisfies the $\left\langle\kappa_{1}\right.$, $\infty$ distribution law.

Proof. From Lemma 2.2 any countable decreasing infinum of base sets is nonzero. The distribution law follows in a standard manner from this fact.

We have now nearly completed the proof of Theorem 2.1. By Lemmas 1.2 and 2.7 there is an ultrafilter $\mu$ on $B$ with $\Pi \mathscr{U}_{i} / D \circ \mu^{(\phi)}=\mu\left(\Pi \mathscr{R}_{i} / D^{(\phi)}\right)$ for every 
sentence $\phi$. Then every sentence true in $B$ has value one in $B^{(B)}$ [3], value one in $\mathfrak{B}^{\prime}$ (Lemma 2.5), value one in $\Pi \mathscr{U}_{i} / D$ (Lemma 2.6) and hence is true in $\Pi \mathscr{R}_{i} / D$ $\circ \mu$ by the above equation. That is to say, $B$ is elementarily equivalent to $\Pi \mathfrak{U}_{i} / D \circ \mu$.

\section{BIBLIOGR APHY ${ }^{(1)}$}

1. F. Galvin, Horn sentences, Ann. Math. Logic 1 (1970), 389-422.

2. H. J. Keisler, Reduced products and Horn classes, Trans. Amer. Math. Soc. 117 (1965), 307-328. MR 30 \#1047.

3. R. Mansfield, The theory of Boolean ultrapowers, Ann. Math. Logic 2 (1971), 297-323.

4. D. Scott and R. M. Solovay, Boolean-valued models of set theory, Proc. Sympos. Pure Math., vol. 13, part 2, Amer. Math. Soc., Providence, R. I. (to appear).

5. S. Shelah, Every two elementarily equivalent models have isomorphic ultrapowers, Israel J. Math. 10 (1971), 224-234.

DEP ARTMENT OF MATHEMATICS, PENNSYLVANIA STATE UNIVERSITY, UNIVERSITY PARK, PENNSYLVANIA 16802 to $[1]$.

(1) For a complete bibliography on the subject of Horn classes, the reader is referred 